\title{
Production of axion-like particles via vector boson fusion at future electron-positron colliders
}

\author{
Chong-Xing Yue ${ }^{\mathrm{a}}$, Hua-Ying Zhang ${ }^{\mathrm{b}}{ }_{\mathbb{D}}$, Han Wang ${ }^{\mathrm{c}}$ \\ Department of Physics, Liaoning Normal University, Dalian 116029, China
}

Received: 28 July 2021 / Accepted: 6 January 2022 / Published online: 29 January 2022

(c) The Author(s) 2022

\begin{abstract}
One kind of particularly interesting pseudoscalar particles, called axion-like particles (ALPs), have rich physical phenomenology at high- and low-energy collider experiments. After discussing most of single production channels of ALP at electron-positron colliders, we investigate the possibility of detecting this kind of new particles through the $\mathrm{W}^{+} \mathrm{W}^{-}$fusion process $\mathrm{e}^{+} \mathrm{e}^{-} \rightarrow \bar{v}_{e} v_{e} a(\rightarrow \gamma \gamma)$ at the CLIC. The $3 \sigma$ and $5 \sigma$ bounds on the ALP parameter space at the three energy stages of the CLIC are obtained. We find that the bounds given by the CLIC are complementary to the existing experiments exclusion regions.
\end{abstract}

\section{Introduction}

After discovery of the Higgs boson in the CMS and ATLAS experiments [1,2], experiments at the LHC have made great achievements and demonstrated that the standard model (SM) is a correct model to explain most of observed phenomena at the electroweak scale. However, deviations of experimental measurements from the SM predictions at or below the percent level are beyond the achievable precision at the LHC and is expected to be performed at the planned $\mathrm{e}^{+} \mathrm{e}^{-}$ colliders with high luminosity and energy. It is well known that, compared to the LHC, the future $\mathrm{e}^{+} \mathrm{e}^{-}$colliders, such as the ILC [3-7], CLIC [8,9], FCC-ee [10,11], and CEPC [12-14] have higher luminosity and more clean experimental environment, which can not only study the SM observables at unprecedented accuracy, but would be very useful to discover the evidence of new physics beyond the SM.

The discovery of the Higgs boson has stimulated interest in searching for additional (pseudo) scalar particles in highand low-energy experiments. Axion-like particles (ALPs) are

\footnotetext{
a e-mail: cxyue@lnnu.edu.cn

b e-mail: huayingheplnnu@163.com (corresponding author)

ce-mail: wangwanghan1106@163.com
}

pseudoscalar bosons that naturally appear in many extensions of the SM as pseudo Nambu-Goldstone bosons arising in explicit global symmetry breaking [15-21]. The properties of ALPs, such as their masses and coupling strengths to the SM particles, are model-dependent and have been extensively investigated. High-energy collider experiments provide possibilities to explore ALPs with masses about $M_{a} \geq$ $1 \mathrm{GeV}$ [22-32]. The constraints on a wide range of the ALP parameter space are obtained using the available data, such as the LEP, Tevatron and LHC data, the future prospects of discovering ALPs at running or future collider experiments are studied. For instance, searches for ALPs via photon fusion are investigated at the $\mathrm{LHC}$ and $\mathrm{e}^{+} \mathrm{e}^{-}$colliders [33-41]. The capabilities of these colliders to probe the ALP parameter space are discussed and the expected bounds on the ALPphoton coupling for a wide range of masses are obtained.

ALPs can also be produced via massive vector boson fusion $(\mathrm{VBF})$ processes (i.e. $\mathrm{W}^{+} \mathrm{W}^{-}$-fusion and $\mathrm{ZZ}$-fusion processes) at high-energy $\mathrm{e}^{+} \mathrm{e}^{-}$colliders [42]. Since the production cross sections of these processes depend logarithmically on the center-of-mass (c.m.) energy, and are generally smaller than those of other processes at low-energy $\mathrm{e}^{+} \mathrm{e}^{-}$ colliders, there are very few studies about ALP productions via $\mathrm{VBF}$ processes with $\mathrm{V}=\mathrm{W}^{ \pm}$, $\mathrm{Z}$. In this work, we will consider the possibilities of detecting ALPs via VBF processes at high-energy $\mathrm{e}^{+} \mathrm{e}^{-}$colliders like the CLIC. We will focus on ALPs that only couple to the electroweak gauge bosons and perform a careful investigation of the ALP signals at high-energy $\mathrm{e}^{+} \mathrm{e}^{-}$colliders from VBF processes. The relevant Feynman diagrams are shown in Fig. 1. Our numerical results show that VBF processes can extend the $\mathrm{e}^{+} \mathrm{e}^{-}$ sensitivity to a region of the ALP parameter space that is not covered by other experiments.

The rest of this paper is organized as follows. After summarizing the effective description of ALP interactions with the electroweak gauge bosons, we calculate and compare the production cross sections of ALPs via $\mathrm{e}^{+} \mathrm{e}^{-}$annihilation 
and VBF processes at $\mathrm{e}^{+} \mathrm{e}^{-}$colliders in Sect. 2. Based on the details of the analysis of the ALP signals and the relevant SM backgrounds from $\mathrm{W}^{+} \mathrm{W}^{-}$fusion process, sensitivity projections of the CLIC to the ALP parameter space are presented in Sect. 3. We report our conclusions in Sect. 4.

\section{Single production of ALP at $\mathrm{e}^{+} \mathrm{e}^{-}$colliders}

The ALP interactions with the SM fermions and gauge bosons arise through five-dimensional operators, and their masses can be treated independently of their couplings [42], which can be described via an effective Lagrangian. The effective interactions of ALP with electroweak gauge bosons, which are related to our calculation, are given by the following dimension-5 effective Lagrangian [27]:

$$
\begin{aligned}
\mathcal{L}_{\text {eff }}^{D \leq 5}= & \frac{1}{2}\left(\partial_{\mu} a\right)\left(\partial^{\mu} a\right)-\frac{M_{a}^{2}}{2} a^{2}+C_{W W} \frac{a}{f_{a}} W_{\mu \nu}^{A} \tilde{W}^{\mu \nu, A} \\
& +C_{B B} \frac{a}{f_{a}} B_{\mu \nu} \tilde{B}^{\mu \nu},
\end{aligned}
$$

where $X_{\mu \nu}$ denotes the field strength tensor for $S U(2)_{L}$ or $U(1)_{Y}, \tilde{X}^{\mu \nu}=\frac{1}{2} \varepsilon^{\mu \nu \alpha \beta} X_{\alpha \beta}$ with $\varepsilon^{0123}=1$ and $X \in\{B, W\}$. The ALP field and mass are denoted by $a$ and $M_{a}$, respectively. The interaction of the ALP with two photons is contributed by the last two terms of Eq.1. The dimensionful couplings $g_{a \gamma \gamma}, g_{a W W}, g_{a \gamma Z}$ and $g_{a Z Z}$, control the coupling strength of the interactions between ALP and electroweak gauge bosons $\left(W^{ \pm}, \mathrm{Z}, \gamma\right)$, which are given by

$$
\begin{aligned}
g_{a \gamma \gamma} & =\frac{4}{f_{a}}\left(c_{\theta_{W}}^{2} C_{B B}+s_{\theta_{W}}^{2} C_{W W}\right), \\
g_{a W W} & =\frac{4}{f_{a}} C_{W W}, \\
g_{a Z Z} & =\frac{4}{f_{a}}\left(s_{\theta_{W}}^{2} C_{B B}+c_{\theta_{W}}^{2} C_{W W}\right), \\
g_{a \gamma Z} & =\frac{4}{f_{a}} s_{2 \theta_{W}}\left(C_{W W}-C_{B B}\right),
\end{aligned}
$$

where $s_{\theta_{W}}=\sin \theta_{W}, c_{\theta_{W}}=\cos \theta_{W}$ with $\theta_{W}$ being the weak mixing angle. To simplify our analysis, we will assume $\mathrm{C}_{W W}$ $=\mathrm{C}_{B B}$ in this work and there are $g_{a \gamma Z}=0, g_{a \gamma \gamma}=g_{a W W}=$ $g_{a Z z} \cdot{ }^{1}$ In this case, ALPs can be singly produced via $\mathrm{e}^{+} \mathrm{e}^{-}$ annihilation and VBF processes at $\mathrm{e}^{+} \mathrm{e}^{-}$colliders. We use FeynRules [44] to generate the UFO model file corresponding to the effective Lagrangian Eq. 1. The cross sections of $\mathrm{e}^{+} \mathrm{e}^{-}$annihilation and VBF processes at $\mathrm{e}^{+} \mathrm{e}^{-}$colliders are calculated, which are shown in Fig. 2. One can see from Fig. 2 that the ALP-strahlung processes ( $\gamma a$ and $Z a$ ) are the main production channels. However, their cross sections become independent of $s$ in the high-energy limit $M_{a}^{2} \ll s$ and thus

\footnotetext{
${ }^{1}$ In this case, Ref. [43] has investigated searching for ALPs via ALPstrahlung process at the LHC.
}

saturate quite soon and flatten out. The cross sections of VBF processes depend only logarithmically on $s$. Therefore, the cross section of $\mathrm{W}^{+} \mathrm{W}^{-}$fusion process grows with $s$ increasing and can reach sizable values at high energies. In principle, the cross section of the $Z Z$ fusion process follows the same trend as that of the WW fusion process, while its value is about an order of magnitude smaller than that of the WW fusion process, due to the fact that the neutral current couplings are smaller than the charged current couplings. The $\gamma \gamma$ fusion process has been extensively discussed in Ref. [39]. The cross section of the ZZ fusion process $\mathrm{e}^{+} \mathrm{e}^{-} \rightarrow$ $\mathrm{e}^{+} \mathrm{e}^{-} a$ is the smallest. In Ref. [45], the similar conclusion is given by exploring the mass and cross section of Higgs with ultimate precision at the FCC-ee.

ALPs have been investigated through the ALP-strahlung processes $\mathrm{e}^{+} \mathrm{e}^{-} \rightarrow V a(a \rightarrow \gamma \gamma)$, where $V=\gamma, Z$ [23,46,47]. In Ref. [23], the ALP-strahlung processes have also been studied at the CLIC, but the author assumes $C_{W W}=$ 0 , considering $\gamma Z a$ coupling, which is opposite to our work. There are previous works about the ALP single production induced by two-photon fusion in future electron-positron colliders [37-39]. Thus, in this paper, we only consider the production of ALP via $\mathrm{W}^{+} \mathrm{W}^{-}$fusion process $\mathrm{e}^{+} \mathrm{e}^{-} \rightarrow \bar{v}_{e} v_{e} a$ with $a$ subsequently decaying to photon pairs (see Fig. 3).

\section{Search for ALP at the CLIC}

As we know, the high-energy $\mathrm{e}^{+} \mathrm{e}^{-}$collider can provide clean experimental environment to explore new physics beyond the Standard Model (BSM). The future $\mathrm{e}^{+} \mathrm{e}^{-}$colliders are contemplated to study the properties of the SM observables with an unprecedented precision, and the CLIC is one of them. The CLIC is planned to be built and operated in three energy stages. The first stage is planned to accurately measure the properties of the Higgs boson at $\sqrt{s}=380 \mathrm{GeV}$ with $\mathcal{L}$ $=0.5 \mathrm{ab}^{-1}$. The second and ultimate stages running at $\sqrt{s}=$ $1500 \mathrm{GeV}$ with $\mathcal{L}=1.5 \mathrm{ab}^{-1}$ and $\sqrt{s}=3000 \mathrm{GeV}$ with $\mathcal{L}=3$ $\mathrm{ab}^{-1}$ are not only able to measure the top Yukawa coupling, the Higgs trilinear self-coupling and the Higgs rare decays, but also can give indirect sensitivity to many new physics scenarios through precision measurements [48]. They will be able to discover new particles through direct detection. We will do calculations and analysis of the signal and background for the process $e^{+} e^{-} \rightarrow \bar{v}_{e} v_{e} a(\rightarrow \gamma \gamma)$ at the CLIC with three energy stages.

In this section, we perform the Monte Carlo (MC) simulation to analyze the possibility of exploring ALPs at the CLIC through the $\mathrm{W}^{+} \mathrm{W}^{-}$fusion process $\mathrm{e}^{+} \mathrm{e}^{-} \rightarrow \bar{v}_{e} v_{e} a$ $(\rightarrow \gamma \gamma)$, where the gauge bosons $\mathrm{W}^{ \pm}$are emitted from the incoming electron/positron beams. Its signal is characterised by two photons, missing transverse energy from the escaping undetected two neutrinos $\left(\bar{v}_{e} v_{e}\right)$. In our numerical calcula- 


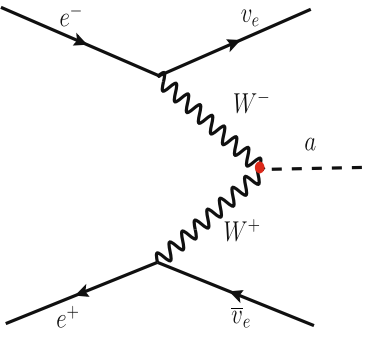

(a)

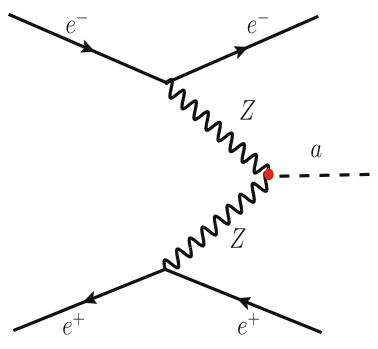

(b)

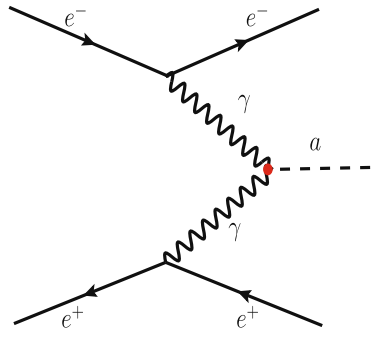

(c)

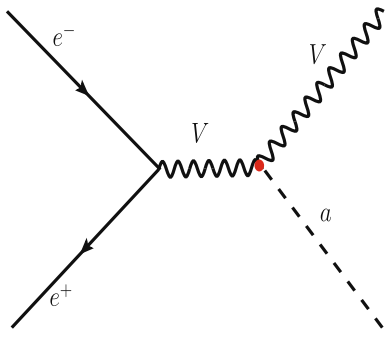

(d)

Fig. 1 The Feynman diagrams of the single production of ALP with $V=\gamma, \mathrm{Z}$ at $\mathrm{e}^{+} \mathrm{e}^{-}$colliders
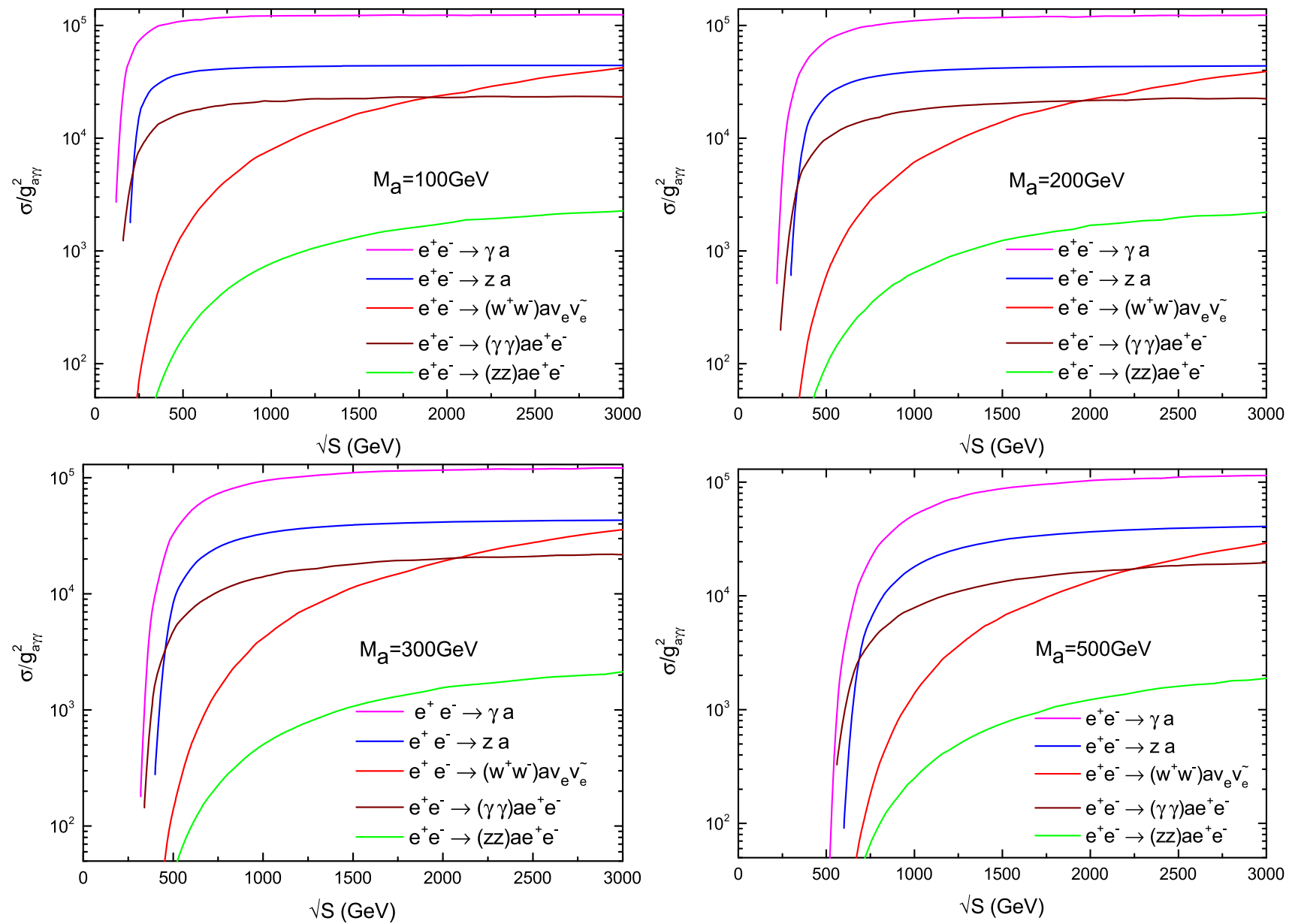

Fig. 2 The ratio $\sigma /\left(g_{a \gamma \gamma}\right)^{2}$ as a function of the c.m. energy $\sqrt{s}$ for $M_{a}=100,200,300$ and $500 \mathrm{GeV}$

tions, we take into account the process $\mathrm{e}^{+} \mathrm{e}^{-} \rightarrow \bar{v} v \gamma \gamma$ as the dominant SM background, which is mainly induced through electroweak interaction. For the signal, the final two photons mainly come from the decay of pseudo-scalar ALPs (spin0 ), while there is no particle with spin-0 in the background that decays to two photons at tree level. So the interference is very small. We have estimated the cross section of pure ALP signal to be $17.25 \mathrm{fb}$ and the cross section of the inter- ference to be $-0.1549 \mathrm{fb}$ at $\sqrt{s}=1500 \mathrm{GeV}, M_{a}=10 \mathrm{GeV}$ and $g_{a \gamma \gamma}=10^{-3} \mathrm{GeV}^{-1}$ without any cut applied. Apparently the cross section of pure ALP signal is two orders of magnitude larger than that of the interference. We have estimated the cross sections of pure ALP signal and interference for multiple ALP benchmark points, and the conclusions are similar. For simplicity, we have neglected the influence of the 


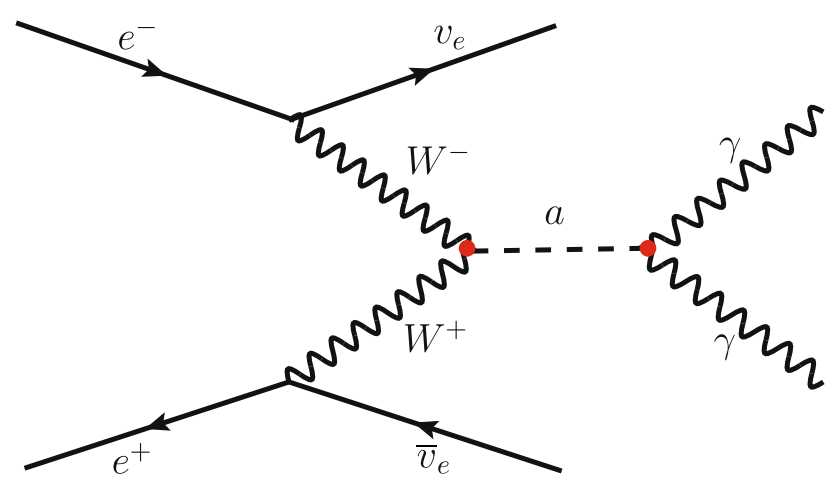

Fig. 3 Feynman diagram of the $\mathrm{W}^{+} \mathrm{W}^{-}$fusion process $\mathrm{e}^{+} \mathrm{e}^{-} \rightarrow \bar{v}_{e} v_{e} a$ $(\rightarrow \gamma \gamma)$

interference between ALP production and SM background in $e^{+} e^{-} \rightarrow \bar{v} \nu \gamma \gamma$.

We look for photons reconstructed in the barrel region $\left|\eta_{\gamma}\right|<2.5$. A light ALP tends to decay a pair of collimated photons, which appear essentially as one photon of the combined energy in a detector and thus can not be correctly reconstructed as two individual photons [49]. In Ref. [46], the light ALP is studied via the decay $Z \rightarrow \gamma a(\rightarrow \gamma \gamma)$ with $\Delta R(\gamma, \gamma) \sim 4 \frac{m_{a}}{m_{Z}} . \Delta R(\gamma, \gamma)=\sqrt{(\Delta \phi)^{2}+(\Delta \eta)^{2}}$ is the particle separation in the rapidity-azimuth plane, $\Delta \phi$ and $\Delta \eta$ are the azimuth difference and pseudo-rapidity difference of the photon pair, respectively. To reconstruct collimated pair of photons from the light ALP decay, a peak separation of $\Delta R(\gamma, \gamma)=4 \frac{m_{\pi}}{E_{\pi}}=0.035$ is taken in Ref. [50], where $m_{\pi}$ and $E_{\pi}$ represent the mass and energy of $\pi^{0}$, and we can see that if $\Delta R(\gamma, \gamma)$ is smaller than 0.035 , making photon pair reconstruction challenging. In our work, the peak of the normalized distribution of $\Delta R(\gamma, \gamma)$ is near 0.06 which allows for sufficient separation for both photons in the pair at $M_{a}=5 \mathrm{GeV}$. To better isolate produced photon pairs, we ask for the isolation between the two photons $\Delta R(\gamma, \gamma)>0.06$ and the photon having a minimum transverse momentum of $30 \mathrm{GeV}$. Based on the above analysis, the basic cuts are chosen as follows:

$\left|\eta_{\gamma}\right|<2.5, \quad \Delta R(\gamma, \gamma)>0.06, \quad p_{T}^{\gamma}>30 \mathrm{GeV}$.

In the following, the signal and background events are generated at tree-level by Madgraph5-aMC@NLO [51] with the above basic cuts. The fast detector simulation are performed with Delphes [52] using the CLIC detector card. Finally, we use MadAnalysis5 [53] package to do the kinematic and cut-based analysis with the reconstructed-level events of signal and background. The final states of the signal and background contain invisible neutrino and two photons. For the signal, two photons in the final state from ALP decay could be a powerful trigger, and the angular separation of two photons depends largely on the ALP mass. Therefore, we choose the invariant mass of the photon pair
$M(\gamma \gamma)$, the angle between reconstructed ALP and beam axis $\theta(\gamma \gamma)$, the missing transverse energy $\mathbb{E}_{T}$ associated to the invisible neutrino system, as well as the transverse energy $E_{T}$ associated to the visible particles as observables in the lab frame. The missing transverse energy and transverse energy are defined by $\mathbb{E}_{T}=\left|\sum_{\text {visible particles }} \vec{p}_{T}\right|$ and $E_{T}=\sum_{\text {visible particles }}\left|\vec{p}_{T}\right|$, where $\vec{p}_{T}$ stands for the visible particles transverse momentum.

In Fig. 4, we plot the normalized distributions of $M(\gamma \gamma)$, $\theta(\gamma \gamma), \mathbb{E}_{T}$ and $E_{T}$ for the signal and background with the signal benchmark points $M_{a}=8,10,20,100,200 \mathrm{GeV}$ and the fixed parameter $g_{a \gamma \gamma}=10^{-3} \mathrm{GeV}^{-1}$ for the c.m. energy $\sqrt{s}=380 \mathrm{GeV}$. In Fig. 5 we show the same normalized kinematic distributions for $M_{a}=8,10,20,100,500,1000$ $\mathrm{GeV}$ and $g_{a \gamma \gamma}=10^{-3} \mathrm{GeV}^{-1}$ at $\sqrt{s}=1500 \mathrm{GeV}$, and the same is shown in Fig. 6 for $M_{a}=8,10,20,100,1000,1500$, $2000 \mathrm{GeV}$ and $g_{a \gamma \gamma}=10^{-3} \mathrm{GeV}^{-1}$ at $\sqrt{s}=3000 \mathrm{GeV}$. In Figs. 4, 5 and 6, all the signal and background samples are obtained by after applying the above basic cuts. From Figs. 4, 5 and 6, we can see that, in order to optimize the signal significance, some additional cuts of kinematic distributions are needed. From these figures, we can see that it is not suitable to impose restrictions on the normalized $M(\gamma \gamma)$ distribution, hence we do not put any constraint. According to the behavioral characteristics of the other three distributions, we can further impose the following improved cuts on the signal and background events as shown in Table 1. Of course, we have applied the basic cuts before applying the cuts in Table 1, and the cuts 1,2 and 3 are applied in sequence.

After imposing the cuts for a few representative ALP mass benchmark points, we summarize the cross sections of the signal and background in Tables 2, 3 and 4 for $\sqrt{s}=$ $380 \mathrm{GeV}, 1500 \mathrm{GeV}$ and $3000 \mathrm{GeV}$, respectively. From Tables 2, 3 and 4, after applying the basic cuts we can find that Tables 3 and 4 show very large differences in the cross sections between $M_{a}=8,10$ and $20 \mathrm{GeV}$, while the variations appear to be minimal in Table 2. For the ALP-photon coupling $g_{a \gamma \gamma}=10^{-3} \mathrm{GeV}^{-1}$, the variations in the cross section of signal are minimal in the ALP mass interval 5 $\mathrm{GeV} \sim 20 \mathrm{GeV}$ without cut applied at $\sqrt{s}=380,1500$ and $3000 \mathrm{GeV}$. The peak of the distribution shifts towards smaller $\Delta R(\gamma, \gamma)$ for the larger c.m. energy at same ALP mass, while the range of $\left|\eta_{\gamma}\right|$ for the high c.m. energy is larger than that for the low c.m. energy at same ALP mass. That is to say, for $\sqrt{s}=3 \mathrm{TeV}$, the signal for $M_{a}=8,10$ and $20 \mathrm{GeV}$ lie very close to the signal region boundaries, so the lighter the ALP the more the signal is cut out. Therefore, the effects of the basic cuts on the cross sections of $M_{a}=8,10$ and 20 $\mathrm{GeV}$ at $\sqrt{s}=1500$ and $3000 \mathrm{GeV}$ are greater than those at $\sqrt{s}=380 \mathrm{GeV}$. From these tables, one can see that the background is suppressed very efficiently, while the signal still has good efficiency after imposing all cuts. 
Table 1 The improved cuts on the signal and background events

\begin{tabular}{llll}
\hline Cuts & $\sqrt{s}=380 \mathrm{GeV}$ & $\sqrt{s}=1500 \mathrm{GeV}$ & $\sqrt{s}=3000 \mathrm{GeV}$ \\
\hline Cut-1: Angle between the ALP and the beam axis & $0.6<\theta(\gamma \gamma)<2.6$ & $0.7<\theta(\gamma \gamma)<2.5$ & $0.5<\theta(\gamma \gamma)<2.6$ \\
Cut-2: The missing transverse energy & $\mathbb{E}_{T}>70 \mathrm{GeV}$ & $\mathbb{E}_{T}>150 \mathrm{GeV}$ & $\mathbb{E}_{T}>200 \mathrm{GeV}$ \\
Cut-3: The transverse energy & $E_{T}>85 \mathrm{GeV}$ & $E_{T}>160 \mathrm{GeV}$ & $E_{T}>220 \mathrm{GeV}$ \\
\hline
\end{tabular}

We use the following Poisson formula [54] to estimate the statistical significance (SS) at the CLIC with the luminosities of $0.5 \mathrm{ab}^{-1}, 1.5 \mathrm{ab}^{-1}$ and $3 \mathrm{ab}^{-1}$, respectively,

$S S=\sqrt{2 \mathcal{L}\left[(S+B) \ln \left(1+\frac{S}{B}\right)-S\right]}$

where $\mathcal{L}$ represents the integrated luminosity, $S$ and $B$ respectively represent the effective cross sections of the signal and background after imposing all cuts.

In Fig. 7, we present the $3 \sigma$ and $5 \sigma$ bounds on the ALP parameter space from the $\mathrm{W}^{+} \mathrm{W}^{-}$fusion process $\mathrm{e}^{+} \mathrm{e}^{-} \rightarrow$ $\bar{v}_{e} v_{e} a(\rightarrow \gamma \gamma)$ at the CLIC. The blue, red and green lines
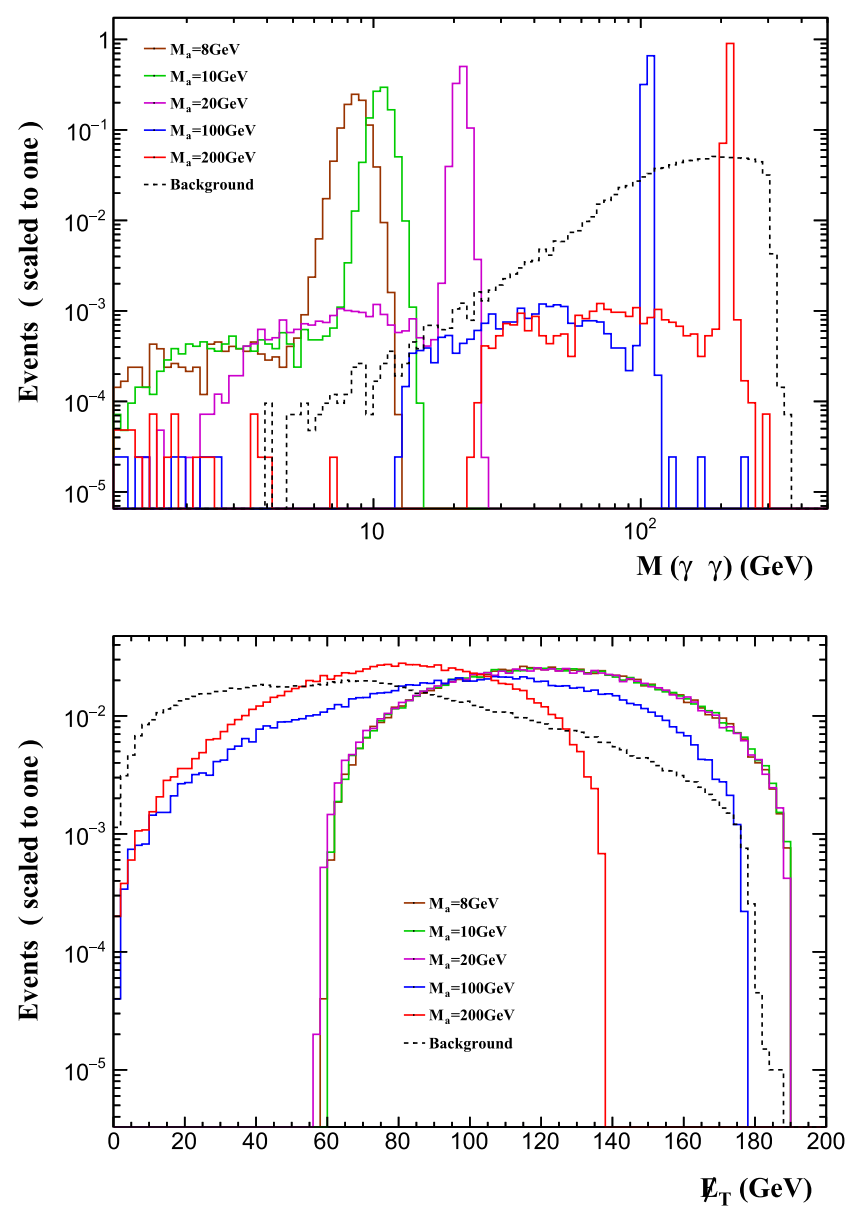

depict the bounds coming from the analysis for the three energy stages of the CLIC. This figure shows that the sensitivity bounds on the ALP-photon coupling $g_{a \gamma \gamma}$ can be improved to $0.8 \mathrm{TeV}^{-1}\left(1.1 \mathrm{TeV}^{-1}\right)$ with the first energy stage, $0.13 \mathrm{TeV}^{-1}\left(0.15 \mathrm{TeV}^{-1}\right)$ with the second energy stage and $0.073 \mathrm{TeV}^{-1}\left(0.091 \mathrm{TeV}^{-1}\right)$ with the third energy stage for the ALP mass interval $5 \mathrm{GeV} \sim 340 \mathrm{GeV}, 5 \mathrm{GeV} \sim$ $1300 \mathrm{GeV}$ and $5 \mathrm{GeV} \sim 2600 \mathrm{GeV}$ at $3 \sigma(5 \sigma)$ levels, respectively. Comparing our results of the three energy stages at the CLIC with each other, it is obvious that the sensitivity bound increases with the c.m. energy increases.
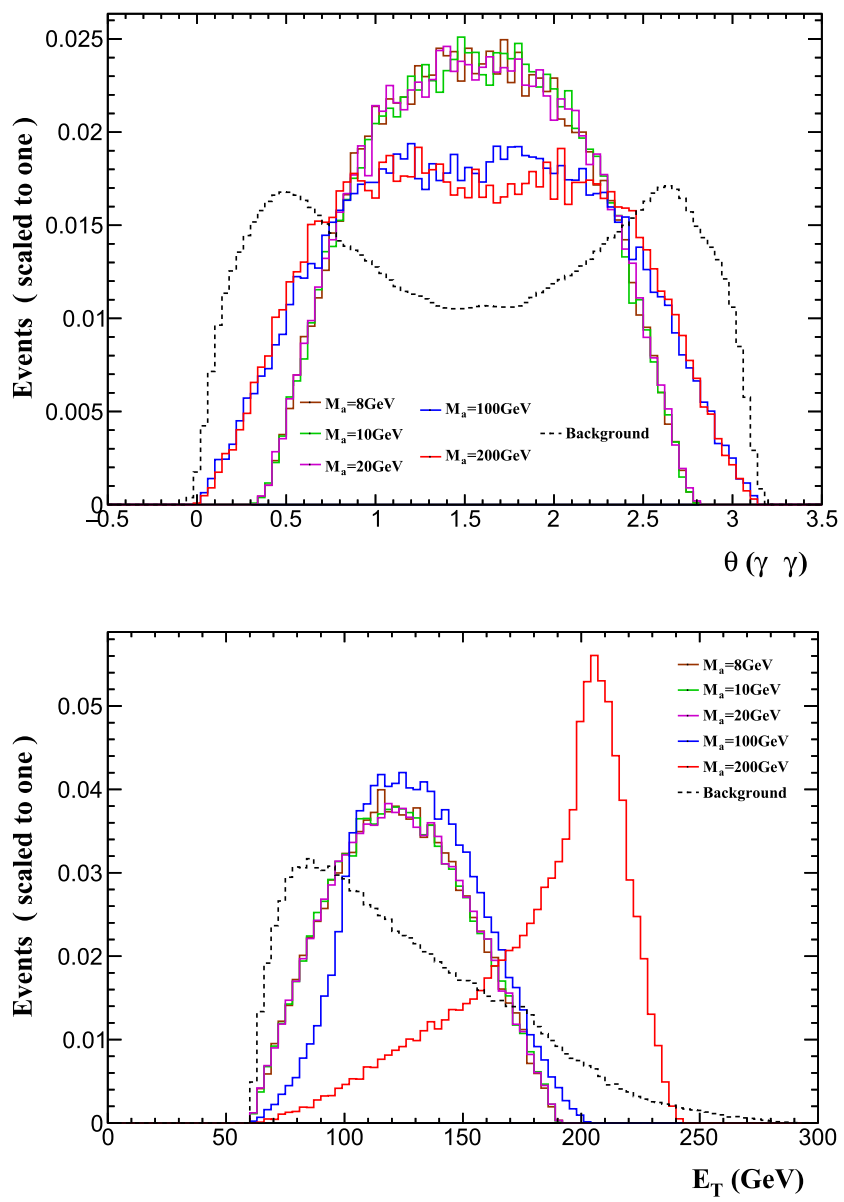

Fig. 4 Normalized distributions of $M(\gamma \gamma), \theta(\gamma \gamma), \mathbb{E}_{T}, E_{T}$ for the signal of selected ALP-mass benchmark points and SM background at the $\sqrt{s}=380 \mathrm{GeV}$ CLIC with designed luminosity 
Table 2 After different cuts applied, the cross sections for the signal and SM background at the $\sqrt{s}=380 \mathrm{GeV}$ CLIC for $M_{a}=8,10,20,100$, $200 \mathrm{GeV}$ and $g_{a \gamma \gamma}=10^{-3} \mathrm{GeV}^{-1}$

\begin{tabular}{|c|c|c|c|c|c|c|}
\hline \multirow[t]{3}{*}{ Cuts } & \multicolumn{6}{|c|}{ CLIC @ $\sqrt{s}=380 \mathrm{GeV}$} \\
\hline & \multicolumn{5}{|l|}{ Signal (fb) } & \multirow{2}{*}{$\begin{array}{l}\text { Background (fb) } \\
\gamma \gamma \bar{\nu} v\end{array}$} \\
\hline & $M_{a}=8 \mathrm{GeV}$ & $M_{a}=10 \mathrm{GeV}$ & $M_{a}=20 \mathrm{GeV}$ & $M_{a}=100 \mathrm{GeV}$ & $M_{a}=200 \mathrm{GeV}$ & \\
\hline Basic cuts & 0.3572 & 0.3542 & 0.3506 & 0.3279 & 0.0731 & 12.66 \\
\hline Cut 1 & 0.3436 & 0.3416 & 0.3364 & 0.2762 & 0.0612 & 7.4612 \\
\hline Cut 2 & 0.3404 & 0.3384 & 0.332 & 0.2464 & 0.0459 & 5.5602 \\
\hline Cut 3 & 0.3198 & 0.318 & 0.3118 & 0.2442 & 0.0457 & 5.3916 \\
\hline
\end{tabular}

Our obtained bounds and other current existing constraints on the ALP-photon coupling are presented in Fig. 8. The green region comes from different beam dump experiments [55-57]. The dark blue and light blue regions depict the results given by $[46,47]$ via the diphoton and triphoton final states $\left(\mathrm{e}^{+} \mathrm{e}^{-} \rightarrow 2 \gamma, 3 \gamma\right)$ at the LEP. The dark gray and black regions denote the constraints from radiative $\Upsilon \rightarrow \gamma a$ at the Babar [58] and the diphoton resonance searches at the $\mathrm{LHCb}$ [59], respectively. The dark green region labelled as "L3" represents looking for isolated and energetic photons from hadronic decay of $\mathrm{Z}$ boson at the L3 collaboration [60]. The exclusion regions also include the results from the LHC resonant $\gamma \gamma$ searches in red $[33,61,62]$. Our projected CLIC sensitivity for $\sqrt{s}=380,1500$ and $3000 \mathrm{GeV}$ are enclosed by the cyan, blue and wine lines, respectively. Comparing our exclusion limits with the results of the LEP and LHC, we can conjecture that the CLIC has better potential to search for ALPs in the lower ALP mass interval $5 \mathrm{GeV} \sim 50 \mathrm{GeV}$ and high ALP mass interval $2 \mathrm{TeV} \sim 2.7 \mathrm{TeV}$.
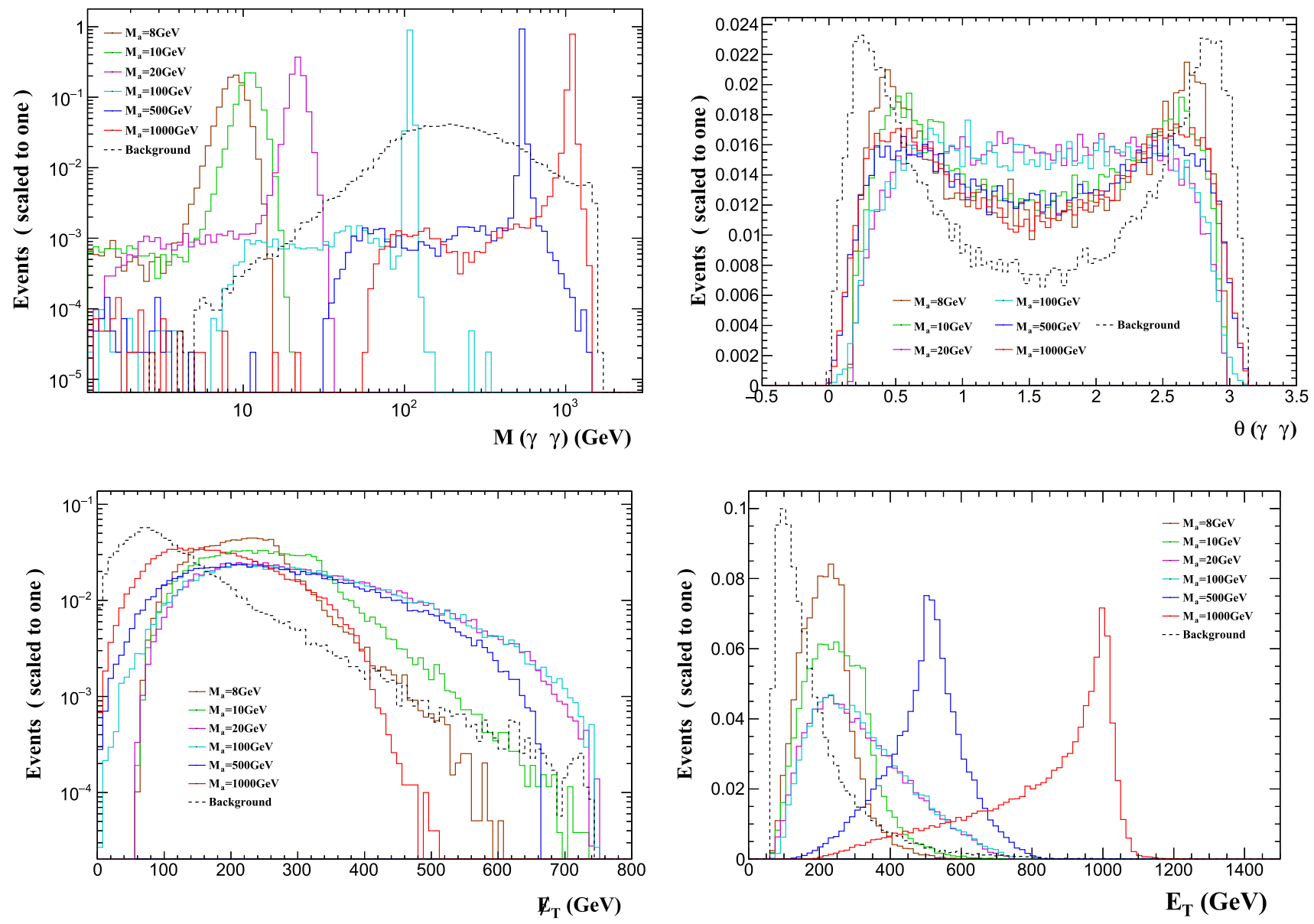

Fig. 5 Same as Fig. 4 but for $\sqrt{s}=1500 \mathrm{GeV}$ CLIC with designed luminosity 

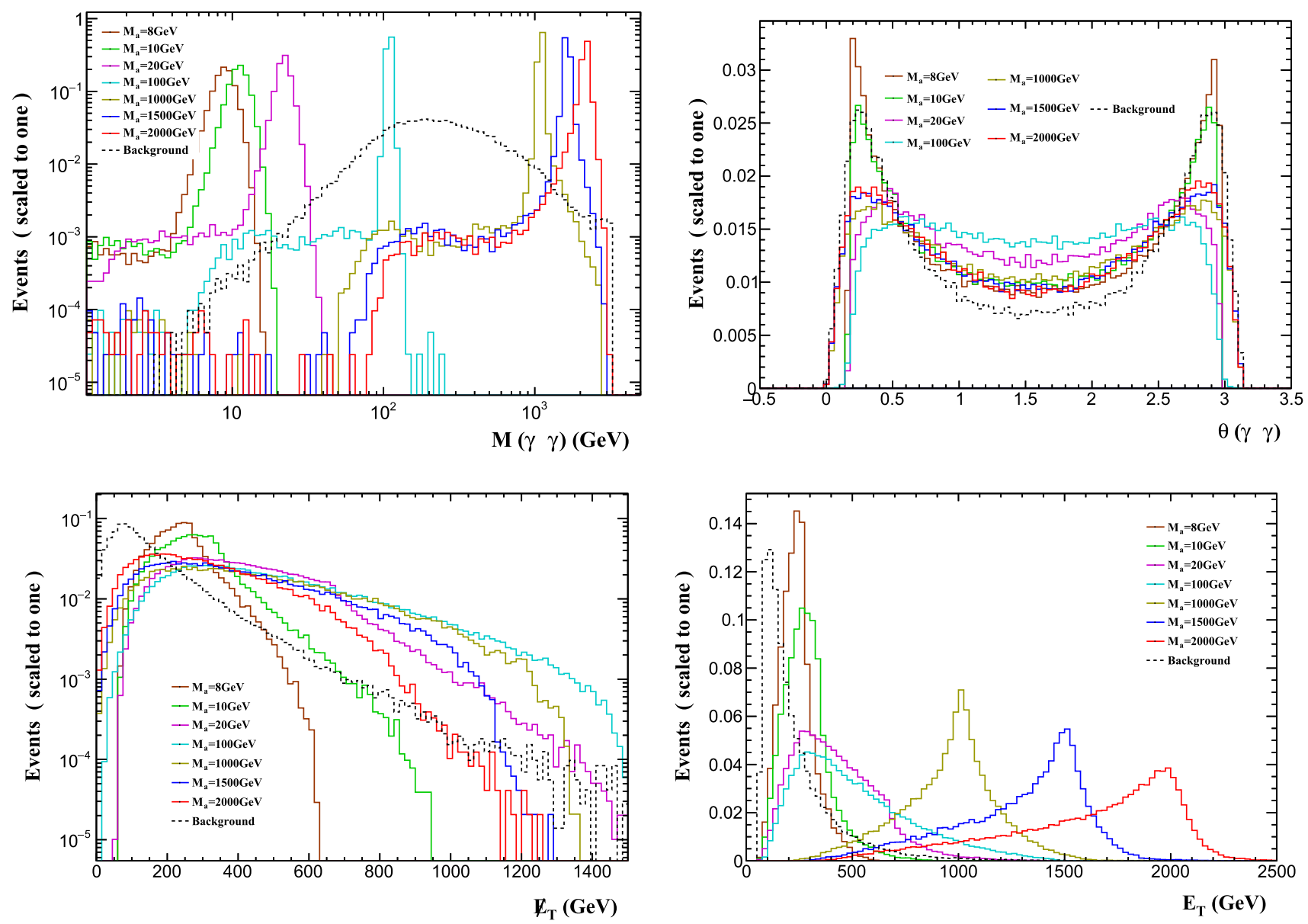

Fig. 6 Same as Fig. 4 but for $\sqrt{s}=3000 \mathrm{GeV}$ CLIC with designed luminosity

Table 3 Same as Table 2 but for the $\sqrt{s}=1500 \mathrm{GeV}$ CLIC

\begin{tabular}{|c|c|c|c|c|c|c|c|}
\hline \multirow[t]{3}{*}{ Cuts } & \multicolumn{7}{|c|}{ CLIC $@ \sqrt{s}=1500 \mathrm{GeV}$} \\
\hline & \multicolumn{6}{|l|}{ Signal $(\mathrm{fb})$} & \multirow{2}{*}{$\begin{array}{l}\text { Background (fb) } \\
\gamma \gamma \bar{\nu} \nu\end{array}$} \\
\hline & $M_{a}=8 \mathrm{GeV}$ & $M_{a}=10 \mathrm{GeV}$ & $M_{a}=20 \mathrm{GeV}$ & $M_{a}=100 \mathrm{GeV}$ & $M_{a}=500 \mathrm{GeV}$ & $M_{a}=1000 \mathrm{GeV}$ & \\
\hline Basic cuts & 6.6575 & 8.8253 & 12.176 & 11.9803 & 1.7862 & 0.1573 & 21.322 \\
\hline Cut 1 & 3.7949 & 5.4839 & 8.6115 & 8.3208 & 1.0745 & 0.0913 & 8.1313 \\
\hline Cut 2 & 3.3201 & 4.9969 & 8.12 & 7.8021 & 1.0991 & 0.0756 & 3.2893 \\
\hline Cut 3 & 3.1913 & 4.8613 & 8.0028 & 7.7769 & 1.0089 & 0.0756 & 3.2211 \\
\hline
\end{tabular}

Table 4 Same as Table 2 but for the $\sqrt{s}=3000 \mathrm{GeV}$ CLIC

\begin{tabular}{|c|c|c|c|c|c|c|c|c|}
\hline \multirow[t]{3}{*}{ Cuts } & \multicolumn{8}{|c|}{ CLIC @ $\sqrt{s}=3000 \mathrm{GeV}$} \\
\hline & \multicolumn{7}{|l|}{ Signal (fb) } & \multirow{2}{*}{$\begin{array}{l}\text { Background (fb) } \\
\gamma \gamma \bar{v} v\end{array}$} \\
\hline & $M_{a}=8 \mathrm{GeV}$ & $M_{a}=10 \mathrm{GeV}$ & $M_{a}=20 \mathrm{GeV}$ & $M_{a}=100 \mathrm{GeV}$ & $M_{a}=1000 \mathrm{GeV}$ & $M_{a}=1500 \mathrm{GeV}$ & $M_{a}=2000 \mathrm{GeV}$ & \\
\hline Basic cuts & 9.6983 & 14.4563 & 27.993 & 32.5997 & 3.5915 & 1.3519 & 0.3323 & 29.1063 \\
\hline Cut 1 & 5.557 & 8.7537 & 20.1513 & 24.8866 & 2.2756 & 0.8178 & 0.2003 & 14.56 \\
\hline Cut 2 & 3.8733 & 7.0693 & 18.4306 & 23.1416 & 2.1683 & 0.7647 & 0.1769 & 5.0493 \\
\hline Cut 3 & 3.3481 & 6.552 & 17.903 & 22.946 & 2.1682 & 0.7647 & 0.1769 & 4.8927 \\
\hline
\end{tabular}




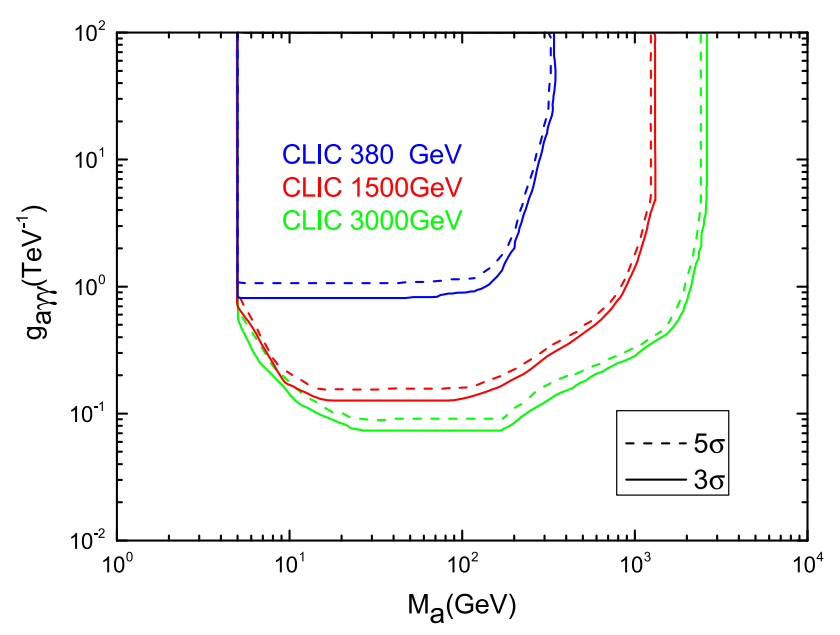

Fig. 7 The $3 \sigma$ and $5 \sigma$ curves in the $M_{a}-g_{a \gamma \gamma}$ plane from the $\mathrm{W}^{+} \mathrm{W}^{-}$ fusion process $\mathrm{e}^{+} \mathrm{e}^{-} \rightarrow \bar{v}_{e} v_{e} a(\rightarrow \gamma \gamma)$ at the 380,1500 and $3000 \mathrm{GeV}$ CLIC with the designed luminosities

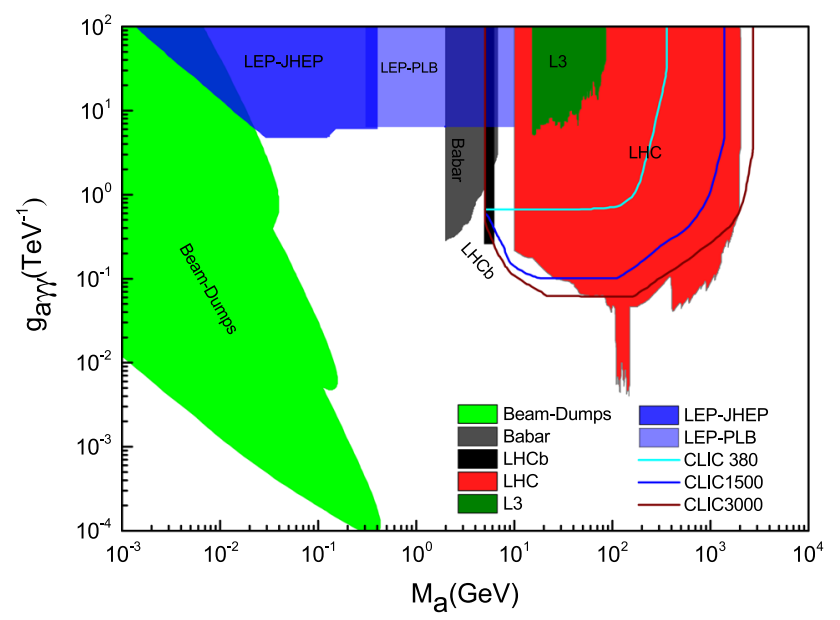

Fig. 8 The $2 \sigma$ exclusion limits on the ALP couplings $g_{a \gamma \gamma}$ as a function of $M_{a}$ from the $\mathrm{W}^{+} \mathrm{W}^{-}$fusion process $\mathrm{e}^{+} \mathrm{e}^{-} \rightarrow \bar{v}_{e} v_{e} a(\rightarrow \gamma \gamma)$ and other current exclusion regions

\section{Conclusions}

As a class of attractive particles, ALPs may influence the structure Electroweak phase transition, play an important role in solving the hierarchy problem and serve as cold dark matter (DM). There are excellent motivations to study ALPs. In this paper, we study the CLIC potential to search for ALP via the $\mathrm{W}^{+} \mathrm{W}^{-}$fusion process $\mathrm{e}^{+} \mathrm{e}^{-} \rightarrow \bar{v}_{e} v_{e} a(\rightarrow \gamma \gamma)$. We have performed the numerical calculations and the phenomenological analysis for the signal and relevant SM background and obtained the $3 \sigma$ and $5 \sigma$ bounds on the ALP parameter space at the three energy stages of CLIC. Comparing our numerical results with other existing bounds, we find that, for the low mass region $5 \mathrm{GeV} \sim 50 \mathrm{GeV}$, the constraint on the ALP-photon coupling $g_{a \gamma \gamma}$ can be improved to $5 \times 10^{-2}$ $\mathrm{TeV}^{-1}$. Meanwhile, our result complements the research of
ALP-photon coupling by the ATLAS and CMS in the ALP mass range of $2 \mathrm{TeV} \sim 2.7 \mathrm{TeV}$.

In conclusion, we have found that searching for ALPs at the CLIC via the $\mathrm{W}^{+} \mathrm{W}^{-}$fusion process $\mathrm{e}^{+} \mathrm{e}^{-} \rightarrow \bar{v}_{e} \nu_{e} a$ $(\rightarrow \gamma \gamma)$ can not only cover low mass ALPs, but also have the greater potential to study the high mass ALPs. Thus, studying ALPs through the $\mathrm{W}^{+} \mathrm{W}^{-}$fusion process $\mathrm{e}^{+} \mathrm{e}^{-} \rightarrow \bar{v}_{e} v_{e} a$ $(\rightarrow \gamma \gamma)$ at the CLIC provides a complementary window to the opportunities offered by other high energy collider experiments.

Acknowledgements This work was supported in part by the National Natural Science Foundation of China under Grants Nos. 11875157 and 12147214

Data Availability Statement This manuscript has no associated data or the data will not be deposited. [Authors' comment: In our paper, Tables 2-4 and Figures 2, 4-8 show our data. Our data were generated by Madgraph5-aMC@NLO. These original data are too large therefore we did not upload them.]

Open Access This article is licensed under a Creative Commons Attribution 4.0 International License, which permits use, sharing, adaptation, distribution and reproduction in any medium or format, as long as you give appropriate credit to the original author(s) and the source, provide a link to the Creative Commons licence, and indicate if changes were made. The images or other third party material in this article are included in the article's Creative Commons licence, unless indicated otherwise in a credit line to the material. If material is not included in the article's Creative Commons licence and your intended use is not permitted by statutory regulation or exceeds the permitted use, you will need to obtain permission directly from the copyright holder. To view a copy of this licence, visit http://creativecomm ons.org/licenses/by/4.0/.

Funded by SCOAP ${ }^{3}$.

\section{References}

1. ATLAS collaboration, G. Aad et al., Observation of a new particle in the search for the Standard Model Higgs boson with the ATLAS detector at the LHC. Phys. Lett. B 716, 1-29 (2012). https://doi. org/10.1016/j.physletb.2012.08.020. arXiv:1207.7214

2. CMS collaboration, S. Chatrchyan et al., Observation of a new boson at a mass of $125 \mathrm{GeV}$ with the CMS experiment at the LHC. Phys. Lett. B 716, 30-61 (2012). https://doi.org/10.1016/ j.physletb.2012.08.021. arXiv:1207.7235

3. ILC collaboration, G. Aarons et al., international linear collider reference design report volume 2: physics at the ILC. arXiv:0709.1893

4. The international linear collider technical design report-volume 2: physics. arXiv: 1306.6352

5. A. Arbey et al., Physics at the e+ e- linear collider. Eur. Phys. J. C 75, 371 (2015). https://doi.org/10.1140/epjc/s10052-015-3511-9 arXiv: 1504.01726

6. ILC collaboration, H. Aihara et al., The international linear collider. A global project. arXiv:1901.09829

7. P. Bambade et al., The international linear collider: a global project. arXiv:1903.01629

8. CLIC Physics Working Group collaboration, E. Accomando et al., Physics at the CLIC multi-TeV linear collider, in 11th international conference on hadron spectroscopy, CERN Yellow Reports: 
Monographs, p. 6 (2004). arXiv:hep-ph/0412251. https://doi.org/ 10.5170/CERN-2004-005

9. CLIC, CLICdp collaboration, The compact linear $\mathrm{e}^{+} \mathrm{e}^{-}$collider (CLIC): physics potential. arXiv:1812.07986

10. TLEP Design Study Working Group collaboration, M. Bicer et al., First look at the physics case of TLEP. JHEP 01, 164 (2014). https:// doi.org/10.1007/JHEP01(2014)164. arXiv:1308.6176

11. FCC collaboration, A. Abada et al., FCC-ee: the lepton collider: future circular collider conceptual design report volume 2. Eur. Phys. J. ST 228, 261-623 (2019).https://doi.org/10.1140/epjst/ e2019-900045-4

12. CEPC Study Group collaboration, CEPC conceptual design report: volume 1-accelerator. arXiv:1809.00285

13. CEPC Study Group collaboration, M. Dong et al., CEPC conceptual design report: volume 2-physics \& detector. arXiv: 1811.10545

14. F. An et al., Precision Higgs physics at the CEPC. Chin. Phys. C 43, 043002 (2019). https://doi.org/10.1088/1674-1137/43/4/043002 arXiv: 1810.09037

15. R.D. Peccei, H.R. Quinn, CP conservation in the presence of instantons. Phys. Rev. Lett. 38, 1440-1443 (1977). https://doi.org/10. 1103/PhysRevLett.38.1440

16. S. Weinberg, A new light boson? Phys. Rev. Lett. 40, 223-226 (1978). https://doi.org/10.1103/PhysRevLett.40.223

17. F. Wilczek, Problem of strong $P$ and $T$ invariance in the presence of instantons. Phys. Rev. Lett. 40, 279-282 (1978). https://doi.org/ 10.1103/PhysRevLett.40.279

18. J.E. Kim, Weak interaction singlet and strong CP invariance. Phys. Rev. Lett. 43, 103 (1979). https://doi.org/10.1103/PhysRevLett.43. 103

19. M.A. Shifman, A. Vainshtein, V.I. Zakharov, Nucl. Phys. B 166, $493(1980)$

20. M. Dine, W. Fischler, M. Srednicki, Phys. Lett. B 104, 199 (1981)

21. A. Zhitnitsky, Sov. J. Nucl. Phys. 31, 260 (1980)

22. M. Bauer, M. Neubert, A. Thamm, Collider probes of axionlike particles. JHEP 12, 044 (2017). https://doi.org/10.1007/ JHEP12(2017)044 arXiv:1708.00443

23. M. Bauer, M. Heiles, M. Neubert, A. Thamm, Axion-like particles at future colliders. Eur. Phys. J. C 79, 74 (2019). https://doi.org/ 10.1140/epjc/s10052-019-6587-9 arXiv:1808.10323

24. J.-F. Fortin, H.-K. Guo, S.P. Harris, D. Kim, K. Sinha, C. Sun, Axions: from magnetars and neutron star mergers to beam dumps and BECs. Int. J. Mod. Phys. D 30, 2130002 (2021). https://doi. org/10.1142/S0218271821300020 arXiv:2102.12503

25. D. d'Enterria, Collider constraints on axion-like particles, in Workshop on feebly interacting particles, p. 2 (2021). arXiv:2102.08971

26. P. Agrawal et al., Feebly-interacting particles: FIPs 2020 workshop report. Eur. Phys. J. C 81, 1015 (2021). https://doi.org/10.1140/ epjc/s10052-021-09703-7 arXiv:2102.12143

27. I. Brivio, M.B. Gavela, L. Merlo, K. Mimasu, J.M. No, R. del Rey et al., ALPs effective field theory and collider signatures. Eur. Phys. J. C 77, 572 (2017). https://doi.org/10.1140/epjc/ s10052-017-5111-3 arXiv:1701.05379

28. S. Knapen, T. Lin, H.K. Lou, T. Melia, Searching for axionlike particles with ultraperipheral heavy-ion collisions. Phys. Rev. Lett. 118, 171801 (2017). https://doi.org/10.1103/PhysRevLett. 118.171801 arXiv: 1607.06083

29. C. Baldenegro, S. Hassani, C. Royon, L. Schoeffel, Extending the constraint for axion-like particles as resonances at the LHC and laser beam experiments. Phys. Lett. B 795, 339-345 (2019). https:// doi.org/10.1016/j.physletb.2019.06.029 arXiv:1903.04151

30. R.O. Coelho, V.P. Goncalves, D.E. Martins, M.S. Rangel, Production of axionlike particles in $P b P b$ collisions at the LHC, HE-LHC and FCC: a phenomenological analysis. Phys. Lett. B 806, 135512 (2020). https://doi.org/10.1016/j.physletb.2020. 135512 arXiv:2002.06027
31. R.O. Coelho, V.P. Gonçalves, D.E. Martins, M. Rangel, Exclusive and diffractive $\gamma \gamma$ production in $P b P b$ collisions at the LHC, HELHC and FCC. Eur. Phys. J. C 80, 488 (2020). https://doi.org/10. 1140/epjc/s10052-020-8006-7 arXiv:2002.03902

32. V.P. Goncalves, D.E. Martins, M.S. Rangel, Searching for axionlike particles with low masses in $\mathrm{pPb}$ and $\mathrm{PbPb}$ collisions. Eur. Phys. J. C 81, 522 (2021). https://doi.org/10.1140/epjc/s10052-021-09314-2 arXiv:2103.01862

33. J. Jaeckel, M. Jankowiak, M. Spannowsky, LHC probes the hidden sector. Phys. Dark Univ. 2, 111-117 (2013). https://doi.org/10. 1016/j.dark.2013.06.001 arXiv:1212.3620

34. C. Baldenegro, S. Fichet, G. von Gersdorff, C. Royon, Searching for axion-like particles with proton tagging at the LHC. JHEP 06, 131 (2018). https://doi.org/10.1007/JHEP06(2018)131 arXiv: 1803.10835

35. A. Flórez, A. Gurrola, W. Johns, P. Sheldon, E. Sheridan, K. Sinha et al., Probing axionlike particles with $\gamma \gamma$ final states from vector boson fusion processes at the LHC. Phys. Rev. D 103, 095001 (2021). https://doi.org/10.1103/PhysRevD.103. 095001 arXiv:2101.11119

36. W.J. Marciano, A. Masiero, P. Paradisi, M. Passera, Contributions of axionlike particles to lepton dipole moments. Phys. Rev. D 94, 115033 (2016). https://doi.org/10.1103/PhysRevD.94.115033 arXiv:1607.01022

37. S.C. İnan, A.V. Kisselev, A search for axion-like particles in lightby-light scattering at the CLIC. JHEP 06, 183 (2020). https://doi. org/10.1007/JHEP06(2020)183 arXiv:2003.01978

38. S.C. İnan, A.V. Kisselev, Polarized light-by-light scattering at the CLIC induced by axion-like particles. Chin. Phys. C 45, 043109 (2021). https://doi.org/10.1088/1674-1137/abe0be arXiv:2007.01693

39. H.-Y. Zhang, C.-X. Yue, Y.-C. Guo, S. Yang, Searching for axionlike particles at future electron-positron colliders. Phys. Rev. D 104, 096008 (2021). https://doi.org/10.1103/PhysRevD.104. 096008 arXiv:2103.05218

40. D. Buttazzo, D. Redigolo, F. Sala, A. Tesi, Fusing vectors into scalars at high energy lepton colliders. JHEP 11, 144 (2018). https://doi.org/10.1007/JHEP11(2018)144 arXiv:1807.04743

41. M.B. Gavela, J.M. No, V. Sanz, J.F. de Trocóniz, Nonresonant searches for axionlike particles at the LHC. Phys. Rev. Lett. 124, 051802 (2020). https://doi.org/10.1103/PhysRevLett.124.051802 arXiv: 1905.12953

42. H. Georgi, D.B. Kaplan, L. Randall, Manifesting the invisible axion at low-energies. Phys. Lett. B 169, 73-78 (1986). https://doi.org/ 10.1016/0370-2693(86)90688-X

43. D. Wang, L. Wu, J.M. Yang, M. Zhang, Photon-jet events as a probe of axionlike particles at the LHC. Phys. Rev. D 104, 095016 (2021). https://doi.org/10.1103/PhysRevD.104.095016 arXiv:2102.01532

44. A. Alloul, N.D. Christensen, C. Degrande, C. Duhr, B. Fuks, FeynRules 2.0-a complete toolbox for tree-level phenomenology. Comput. Phys. Commun. 185, 2250-2300 (2014). https://doi.org/ 10.1016/j.cpc.2014.04.012. arXiv:1310.1921

45. P. Azzurri, G. Bernardi, S. Braibant, D. d'Enterria, J. Eysermans, P. Janot et al., A special Higgs challenge: measuring the mass and production cross section with ultimate precision at FCC-ee. Eur. Phys. J. Plus 137, 23 (2022). https://doi.org/10.1140/epjp/ s13360-021-02202-4 arXiv:2106.15438

46. J. Jaeckel, M. Spannowsky, Probing MeV to $90 \mathrm{GeV}$ axion-like particles with LEP and LHC. Phys. Lett. B 753, 482-487 (2016). https://doi.org/10.1016/j.physletb.2015.12.037 arXiv:1509.00476

47. K. Mimasu, V. Sanz, ALPs at colliders. JHEP 06, 173 (2015). https://doi.org/10.1007/JHEP06(2015)173 arXiv:1409.4792

48. CLIC, CLICdp collaboration, M.J. Boland et al., Updated baseline for a staged Compact Linear Collider. arXiv:1608.07537

49. B. Sheff, N. Steinberg, J.D. Wells, Higgs boson decays into narrow diphoton jets and their search strategies at the Large Hadron Col- 
lider. Phys. Rev. D 104, 036009 (2021). https://doi.org/10.1103/ PhysRevD.104.036009 arXiv:2008.10568

50. N. Steinberg, J.D. Wells, Axion-like particles at the ILC Giga-Z. JHEP 08, 120 (2021). https://doi.org/10.1007/JHEP08(2021)120 arXiv:2101.00520

51. J. Alwall, R. Frederix, S. Frixione, V. Hirschi, F. Maltoni, O. Mattelaer et al., The automated computation of tree-level and nextto-leading order differential cross sections, and their matching to parton shower simulations. JHEP 07, 079 (2014). https://doi.org/ 10.1007/JHEP07(2014)079 arXiv:1405.0301

52. DELPHES 3 collaboration, J. de Favereau, C. Delaere, P. Demin, A. Giammanco, V. Lemaître, A. Mertens et al., DELPHES 3, a modular framework for fast simulation of a generic collider experiment. JHEP 02, 057 (2014). https://doi.org/10.1007/JHEP02(2014)057. arXiv: 1307.6346

53. E. Conte, B. Fuks, G. Serret, MadAnalysis 5, a user-friendly framework for collider phenomenology. Comput. Phys. Commun. 184, 222-256 (2013). https://doi.org/10.1016/j.cpc.2012.09.009 arXiv: 1206.1599

54. G. Cowan, K. Cranmer, E. Gross, O. Vitells, Asymptotic formulae for likelihood-based tests of new physics. Eur. Phys. J. C 71, 1554 (2011). https://doi.org/10.1140/epjc/s10052-011-1554-0 arxiv: 1007.1727

55. E.M. Riordan et al., A search for short lived axions in an electron beam dump experiment. Phys. Rev. Lett. 59, 755 (1987). https:// doi.org/10.1103/PhysRevLett.59.755
56. J.D. Bjorken, S. Ecklund, W.R. Nelson, A. Abashian, C. Church, B. Lu et al., Search for neutral metastable penetrating particles produced in the SLAC beam dump. Phys. Rev. D 38, 3375 (1988). https://doi.org/10.1103/PhysRevD.38.3375

57. B. Döbrich, J. Jaeckel, F. Kahlhoefer, A. Ringwald, K. SchmidtHoberg, ALPtraum: ALP production in proton beam dump experiments. JHEP 02, 018 (2016). https://doi.org/10.1007/ JHEP02(2016)018 arXiv:1512.03069

58. BaBar collaboration, J.P. Lees et al., Search for hadronic decays of a light Higgs boson in the radiative decay $\Upsilon \rightarrow \gamma A^{0}$. Phys. Rev. Lett. 107, 221803 (2011). https://doi.org/10.1103/PhysRevLett. 107.221803. arXiv:1108.3549

59. X. Cid Vidal, A. Mariotti, D. Redigolo, F. Sala, K. Tobioka, New axion searches at flavor factories. JHEP 01, 113 (2019). https://doi. org/10.1007/JHEP01(2019)113. arXiv:1810.09452

60. L3 collaboration, O. Adriani et al., Isolated hard photon emission in hadronic Z0 decays. Phys. Lett. B 292, 472-484 (1992).https:// doi.org/10.1016/0370-2693(92)91205-N

61. ATLAS collaboration, G. Aad et al., Measurement of isolatedphoton pair production in $p p$ collisions at $\sqrt{s}=7 \mathrm{TeV}$ with the ATLAS detector. JHEP 01, 086 (2013). https://doi.org/10.1007/ JHEP01(2013)086. arXiv:1211.1913

62. A. Mariotti, D. Redigolo, F. Sala, K. Tobioka, New LHC bound on low-mass diphoton resonances. Phys. Lett. B 783, 13-18 (2018). https://doi.org/10.1016/j.physletb.2018.06.039 arXiv:1710.01743 